\title{
A PRELIMINARY REPORT ON A MULTIPLE-CHANNEL COCHLEAR IMPLANT OPERATION
}

BY

Y. C. TONG, R. C. BLACK, G. M. CLARK, I. C. FORSTER, J. B. MILLAR, B. J. O'LOUGHLIN and J. F. PATRICK

THE ROYAL COLLEGE OF PSYCHIATRISTS

(C) 1979 


\title{
A preliminary report on a multiple-channel cochlear implant operation
}

\author{
By Y. C. Tong, R. C. Black, G. M. Clark, I. C. Forster, J. B. Millar, \\ B. J. O'Loughlin and J. F. PATRICK (Melbourne)
}

\section{Introduction}

INTRA-COCHLEAR single-channel electrical stimulation has recently been attempted by Michelson (1971) and by House and Urban (1973). Douek et al. (1977) have described experiments with a single-channel promontory electrode system. It is generally accepted that a single-channel system is useful in conveying crude auditory information such as the presence of sounds and some prosodic features of speech (Bilger et al., 1977; Douek et al., 1977).

Bilger et al. (1977) have concluded that single-channel electrical stimuli appear to be analogous to acoustic stimuli varying along a single auditory dimension as subjects seem able to identify members of a small set of five to nine stimuli. This small message set is not adequate for the purpose of speech comprehension. Another approach is to use multiplechannel electrical stimulation systems. The rationale is to stimulate selectively small groups of residual auditory nerve fibres with a number of electrodes so that more information about the input acoustic signals may be conveyed to the higher levels of the auditory system. Furthermore, with appropriate placement of electrodes, the tonotopic organization of the auditory nerve might be utilized, and frequency components of the input acoustical signal could be coded in terms of the place principle of hearing.

Multiple-channel electrical stimulation using modiolar electrodes was used by Simmons (1966). Both monopolar and bipolar stimulation was attempted with six implanted modiolar electrodes. The results showed that the pitch sensations produced depended both on the electrode that was activated (described by Simmons as 'characteristic pitch') and the pulse rate of stimulation (described as 'rate pitch'). The subject, however, was unable to identify individual speech sounds.

The disadvantage of the modiolar approach is that the electrode array cannot be located with the precision necessary to allow consistent tonotopic stimulation in different patients. An alternative approach is to make use of the tonotopic organization of the auditory nerve fibres in the cochlea by inserting an electrode array into the scala tympani.

Multiple-channel intra-cochlear electrode systems were implanted and studied by Mladejovsky et al. (1975) and by Chouard and Macleod (1976). Both groups used monopolar electrodes implanted in the scala tympani, 
with the single ground electrode outside the cochlea. The results of the study of Mladejovsky et al. were similar to those of Simmons (1966)pitch was found to be dependent on electrode placement and rate of stimulation. The usefulness of their system for speech communication has yet to be described. Chouard et al. have reported some improvement in lip-reading and articulation for subjects using their electrode and speech processing systems. However, the results of their rehabilitation programme have not yet been reported.

In this paper, we present the results of a series of preliminary experiments conducted with a patient having a multiple-electrode array implanted into the scala tympani (Clark et al., 1979b). The major difference between the present system and previous intra-cochlear monopolar systems (Mladejovsky et al., 1975; Chouard and Macleod, 1976) is that an electrode array with inter-leaving ground and active electrodes is used. Electrical stimulation using such an array reduces the spread of electric current in the scala tympani (Black and Clark, 1977; Black, 1978; Black and Clark, 1978), and therefore reduces the size of the overlapping fibre population stimulated by adjacent electrodes. Furthermore, the electrodes are driven by an implanted receiver-stimulator designed to allow experimentation of different stimulus-coding strategies.

\section{Patient selection and clinical management}

The patient, R.S., who received our first multiple-channel cochlear implant on 1 August 1978 was a 46-year-old male who experienced sudden and total hearing loss on 15 January 1977 following a head injury. He received a severe blow to the back of the head in a car accident, and this was complicated by a left occipital extradural haematoma requiring urgent surgery. Post-operatively he manifested bulbar and facial nerve palsies which gradually improved. Bilateral total hearing loss, tinnitus and loss of balance have persisted since that time.

On 17 April 1978 the patient was first referred for evaluation for a multiple-channel cochlear implant and this was carried out according to the guidelines previously discussed (Clark et al., 1977b). Clinical assessment revealed no previous history of ear disease, and the ears, nose and throat were normal on examination. Apart from essential hypertension there were no other relevant medical findings. Pure-tone and speech audiometry showed no hearing in either ear, and consequently other audiological and psycho-acoustic tests could not be performed. An impedance audiogram demonstrated normal middle ear and Eustachian tube function. The x-rays confirmed the absence of middle ear or mastoid disease, but demonstrated a small crack fracture entering the left superior semicircular canal. Electronystagmography showed no vestibular function on either side.

Electrical stimulation of the promontory was performed on both ears 
under local anaesthetic. Stimulation was carried out with trains of monophasic rectangular current pulses with a pulse width of $200 \mu \mathrm{s}$ and a duration of $300 \mathrm{~ms}$. These pulse trains were presented at a rate of $1-2 / \mathrm{s}$. Ascending and descending threshold currents were determined for both ears. The patient reported on the left side that he had tonal sensations from $50 \mathrm{pps}$ to $200 \mathrm{pps}$. The perception of pitch as high or low did not show a significant trend with increasingly higher rates of stimulation. On the right side the patient did not initially describe the electrical stimuli as tonal, but commented on them as a 'dash - dash', 'click - click' sensation. With an increase in the rate of stimulation, however, there was an increase in the pitch perceived. In addition the patient also reported that his tinnitus was masked by electrical stimulation of the left ear but not the right.

As the $\mathrm{x}$-rays did not show a fracture in the region of the internal auditory meati but a small one entering the left superior semicircular canal, and as electrical stimulation of the promontory revealed that the patient could experience hearing sensations, it was decided that the injury had not caused transection of the auditory nerves but concussion of the cochlea. For this reason a multiple-channel cochlear implant was recommended, and the left ear was selected as electrical stimulation of the promontory had produced more tonal sensations on this side. A further advantage in selecting this patient was his musical appreciation and previous experience in singing in choirs.

After the risks and expectations of a multiple-channel cochlear implant were explained, the patient was assessed by an Ethics Committee to ensure that all the risks had been adequately explained and no coercion used. This Ethics Committee consisted of one member representing the implant team, an independent otolaryngologist, a clinical psychologist, a practising lawyer and a mother of a deaf child.

On 1 August 1978 a multiple-channel cochlear implant operation was performed on the patient. A ten-channel electrode array was inserted through an opening in the round window membrane for a distance of 25 $\mathrm{mm}$ around the scala tympani. The receiver-stimulator unit was positioned in a bed created in the mastoid bone and held in place by a silastic mould, and superficial and deep fascial flaps (Clark et al., 1979b). The patient made a good recovery and was ambulant in three days, and discharged from hospital in two weeks. Wound healing was satisfactory, and the first electrical stimulation test was performed three weeks after surgery.

A subsequent $\mathrm{x}$-ray of the temporal bone, to evaluate the electrode placement, showed that the proximal or most basal electrode was opposite the round window region. This would indicate that the electrode array had shifted about $10 \mathrm{~mm}$, and emphasizes the need for adequate extra-cochlear fixation.

\section{Electrode array}

The multiple-channel cochlear prosthesis requires an electrode system which produces a stimulus current spatially localized to each electrode. A 
bipolar electrode pair placed in the scala tympani produces significantly less spread of current than with monopolar stimulation of the scala tympani in which the earth electrode is outside the cochlea (Schindler et al., 1977; Black and Clark, 1979). The electrode array used in the present implant does not consist of discrete bipolar pairs, but uses an extended earth system which is common to all stimulus channels. It consists of twenty individual platinum foil bands $0.3 \mathrm{~mm}$ wide and spaced $0.75 \mathrm{~mm}$ apart on a silicon rubber tube of outside diameter $0.4 \mathrm{~mm}$ at the tip, increasing to $0.64 \mathrm{~mm}$ diameter at the point $8 \mathrm{~mm}$ from the tip. Each band is connected to the receiver-stimulator by a $0.025 \mathrm{~mm}$ teflon-coated wire running inside the silicon rubber tube. Ten alternate bands are connected to form the extended earth system, and the remaining bands serve as the ten active electrodes.

Experiments have been performed with such electrodes implanted into the scala tympani of cats. Measurements of the ground current in each earth band electrode during stimulation of the active electrodes showed that even for such an extended ground system, the current attenuation was in the range $2-4.5 \mathrm{~dB} / \mathrm{mm}$. This compares with an attenuation in the range of $0.5-1.0 \mathrm{~dB} / \mathrm{mm}$ for monopolar stimulation of the scala tympani determined from nerve excitability studies (Schindler et al., 1977; Black and Clark, 1979).

\section{System hardware}

The prosthesis system acts as a multiple-channel transcutaneous stimulation system (Forster, 1978; Forster and Patrick, 1978), and may be considered to comprise three components as shown in block diagram form in Fig. 1. The stimulus processor and transmitter stages are both located external to the patient, while the receiver-stimulator was located in a surgically-created bed in the mastoid bone.

The stimulus pattern to be delivered to the intracochlear electrode array described above, is specified by an HP2100 computer system. The

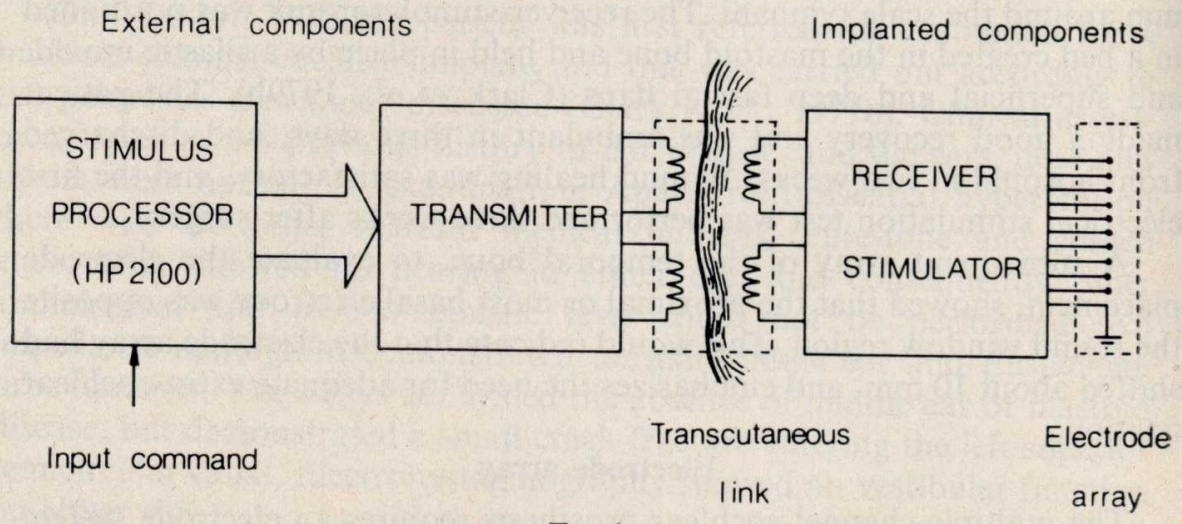

Fig. 1

A block diagram of the overall prosthesis system. 
use of software control thus provides a high degree of overall flexibility with respect to stimulus pattern generation. The second stage, the transmitter, receives the digitally encoded stimulus data from the computer, formats these data and subsequently generates radio frequency power and data signals which are inductively coupled to the implanted receiverstimulator. This third stage decodes the received data, and presents the stimuli to the appropriate electrodes. More detailed descriptions of the design, operation and realization of the transmitter and receiver-stimulator stages are to be found elsewhere (Clark et al., 1977a; Clark et al., 1977c; Clark et al., 1978; Forster, 1978).

The transcutaneous approach, in which no direct connection exists between the intra-cochlear electrode array and the stimulus processor, was chosen for the present system to avoid the risks associated with the alternative, percutaneous approach. In the latter technique, non-biological materials must pass directly through, and make contact with, the skin and underlying tissue (e.g. Simmons, 1966) and this may result in infection, and long term patient trauma. Furthermore, the transcutaneous transfer of power to the receiver-stimulator obviates the need for an implanted power source such as batteries.

The system outlined above permits the independent control of up to ten electrodes, and specifies the amplitude as stimulus current intensity and the time of stimulation or phase. The amplitude parameter permits control of stimulus current in quanta of approximately $67 \mu \mathrm{A}$ up to a maximum of $1 \mathrm{~mA}$, whilst the phase parameter permits a time resolution of $125 \mu \mathrm{s}$. Furthermore, the maximum steady state pulse rate of stimulation is $1,000 \mathrm{pps}$, and the stimulus pulse width is fixed at approximately $180 \mu \mathrm{s}$.

In the present arrangement, the external inductive coupling unit, measuring approximately $23 \mathrm{~mm}$ in diameter and $6 \mathrm{~mm}$ in thickness, is attached to a standard bone conduction aid headband, which is worn by the patient. The external coupling unit is positioned over the corresponding coupling unit of the receiver-stimulator: should the stimulus become painful or irritating, it may be easily removed by the patient, thus rendering the implanted device inoperative. In addition, a remote cut-out facility is incorporated in the hardware system, which the patient can easily activate if necessary.

Finally, it should be noted that facilities have been provided to permit the recording of the scalp potentials generated by stimulus currents. This is carried out with a low-noise, high-gain biological amplifier, together with surface electrodes placed on the lower mastoid and neck, and enables us to verify the performance of the implanted receiver-stimulator.

\section{Results}

Some preliminary studies involving electrical stimulation of the auditory nerve by single electrodes, and by simultaneous activation of two 
electrodes have been performed. The results are presented in two sections. Section A describes the results obtained in four psychophysical experiments designed to reveal the nature of the sensations produced by steady state pulse trains in single electrodes. Section B describes two exploratory studies involving simultaneous activation of two electrodes with steady state pulse trains.

All pulse trains used in these studies consisted of constant current, biphasic pulses. The pulse width of each phase was $180 \mu$ s. Fifteen current levels ranging from $67 \mu \mathrm{A}$ (level 1) to approximately $1 \mathrm{~mA}$ (level 15) could be assigned in $67 \mu \mathrm{A}$ steps. The electrodes were numbered from 0 to 9 according to the physical location of the electrodes. Electrode 0 corresponds to the most apical intra-cochlear electrode, and electrode 9 the most basal one and the closest to the round window region. Initial tests revealed that electrode 0 had a very high threshold, and was not used in subsequent testing. The results presented in this paper were obtained with the remaining nine electrodes (1-9).

\section{(A) Single-electrode studies}

\section{(i) Threshold measurements}

Threshold current levels for the nine active electrodes were determined by an ascending method. Single electrodes were driven by $300 \mathrm{~ms}$ pulse trains that had rise-decay times of $50 \mathrm{~ms}$. Threshold curves for two pulse rates (200 pps and 1,000 pps) are shown in Fig. 2. For all electrodes, the threshold current decreased with an increase in pulse rate.

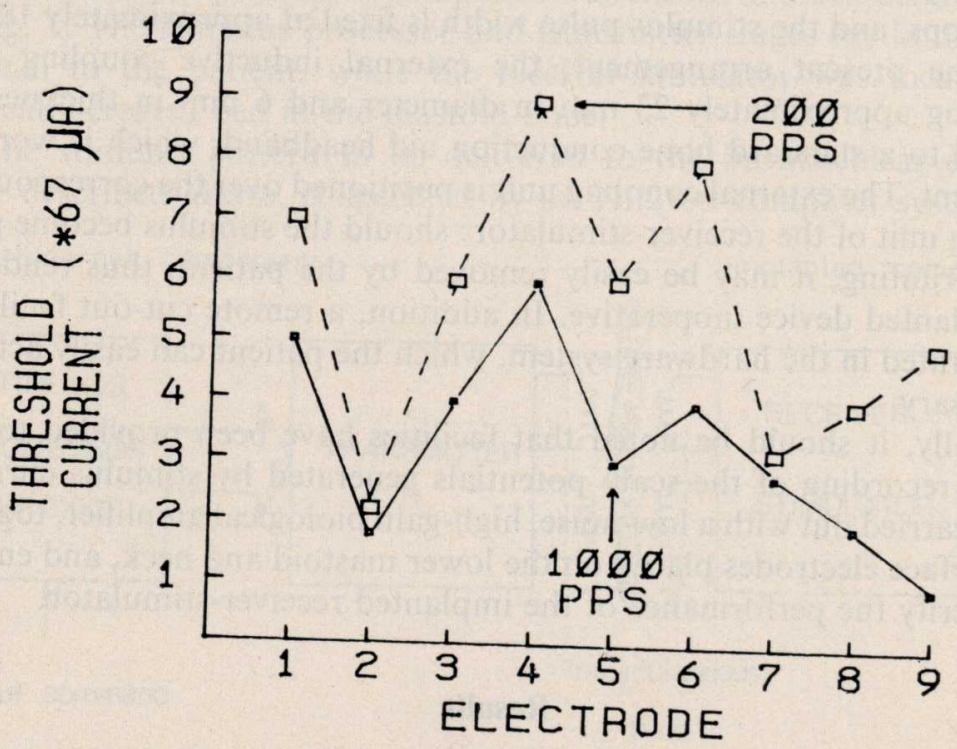

FIG. 2

Threshold currents for the nine electrodes at stimulus rates of $200 \mathrm{pps}$ and $1000 \mathrm{pps}$. 
The variation of threshold currents between the electrodes could be a result of a combination of the following two factors: (i) differences in the densities of surviving nerve fibres about the electrode surfaces, and (ii) differences in the electrical impedances between the active and ground electrodes, and between the active electrodes and the internal auditory meatus.

\section{(ii) Loudness scaling}

The variation of apparent loudness as a function of stimulus current level was determined by a magnitude estimation procedure using no standard (Stevens, 1975). $300 \mathrm{~ms}$ pulse trains with rise-decay times of $50 \mathrm{~ms}$ were delivered to single electrodes in irregular order. The patient was asked to assign numbers estimating the apparent loudnesses produced by the pulse trains. Figure 3 shows the magnitude estimates for two

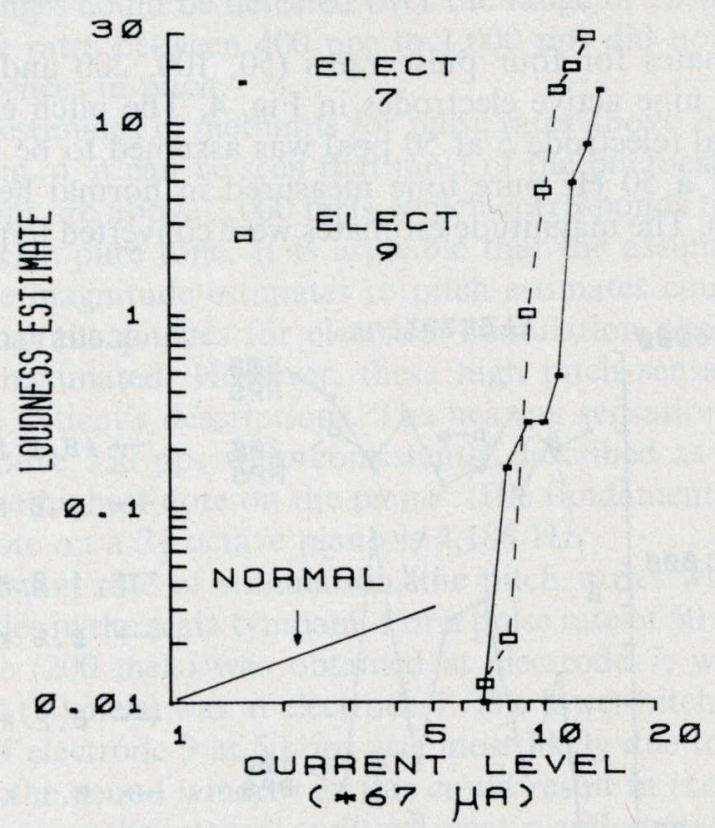

FIG. 3

Loudness magnitude estimates for different current levels when stimulating electrodes 7 and 9 at 200 pps.

representative electrodes driven by 200 pps pulse trains. Each data point in the log-log plot is the mean of two estimates at each current level. The loudness growth characteristics for normal hearing subjects are also shown in the figure for comparison.

For both electrodes, the loudness growth due to increases in current level was much steeper than the growth for acoustical stimulation in normal hearing subjects. This steep growth of sensory magnitude was also observed in the electrical stimulation of the skin. The growth of sensory magnitude 
for electrical stimulation of the skin was found to be much steeper than that for mechanical stimulation (Teghtsoonian, 1971; Stevens, 1975).

(iii) Pitch scaling

The variation of pitch as a function of pulse rate and electrode position was investigated by a magnitude estimation procedure. The stimuli delivered to single electrodes were $300 \mathrm{~ms}$ pulse trains with rise-decay times of $50 \mathrm{~ms}$. The patient was presented with two $300 \mathrm{~ms}$ pulse trains (signals) separated in time by a $1 \mathrm{~s}$ interval, and was asked to assign a number estimating the number of times the second signal was higher or lower in pitch than the first. A reference stimulus, 50 pps pulse train at electrode 5 , was always presented as the first signal. For an individual electrode, a fixed current level was used for all pulse rates. The current levels for the electrodes were obtained by balancing the loudnesses of 200 pps stimuli across electrodes.

Pitch estimates for four pulse rates $(50,100,200$ and $500 \mathrm{pps})$ are shown for the nine active electrodes in Fig. 4 . The pitch estimate of the reference signal (electrode 5 at 50 pps) was assumed to be 78 mels which is the pitch of a $50 \mathrm{~Hz}$ pure tone measured in normal hearing subjects (Stevens, 1975). The magnitude estimates were converted to pitch estimates

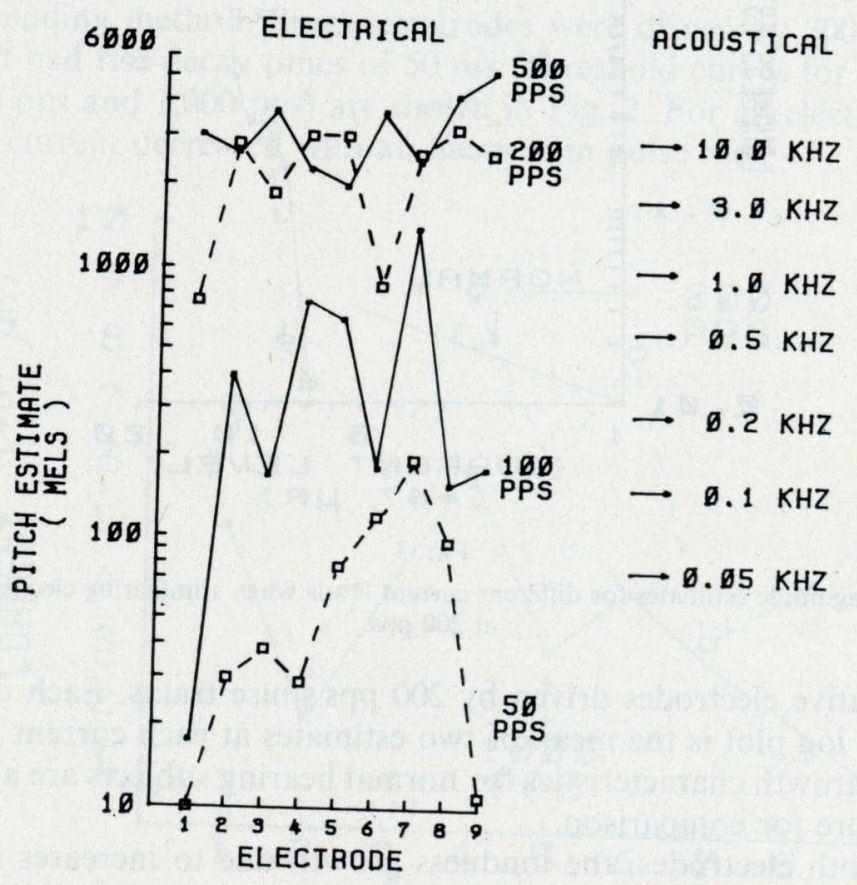

FIG. 4

Pitch estimates in mels for the nine electrodes when stimulating at rates of 50, 100, 200 and 500 pps. 
in mel-units with respect to the reference. Each data point in Fig. 4 is the mean of two estimates. The pitch estimates (mel-units) for pure tones at seven frequencies measured in normal hearing subjects are also shown in Fig. 4 for comparison.

For pulse rates below $200 \mathrm{pps}$, the pitch produced by electrical stimulation increases with pulse rate. For example, the pitch estimate for electrode 5 increased from 78 mels at 50 pps to 3,000 mels at 200 pps. However, for pulse rates above $200 \mathrm{pps}$, the increase of pitch with pulse rate is not as pronounced. As shown in Fig. 4 there is a considerable overlap of pitch estimates at 200 pps and 500 pps.

The variation of pitch with pulse rates described here is similar to the result reported by Mladejovsky et al. (1975). For monopolar stimulation using biphasic current pulses, Mladejovsky et al., reported that although pulse rate changes could be detected over the range of $25-400 \mathrm{pps}$, stimulation at pulse rates between $400 \mathrm{pps}$ to $1,000 \mathrm{pps}$ did not result in perceivable differences in pitch.

The pitch estimates in mel-units for pulse rates above 200 pps are very high. From Fig. 4, it can be seen that most of the pitch estimates for 200 pps and 500 pps are above 2,000 mels, which corresponds to the perceived pitch of a $3 \mathrm{kHz}$ pure tone. It is arguable that the assumption made in converting the magnitude estimates to pitch estimates could be inappropriate, and that the pitches for electrical stimulation above $200 \mathrm{pps}$ are therefore overestimated. However, these high pitch sensations are confirmed by the patient's descriptions. The hearing sensations produced by pulse rates above $250 \mathrm{pps}$ were consistently described as having pitches 'way above the highest note on the piano'. The fundamental frequency of the highest note on a $7 \frac{1}{4}$ octave piano is $4,186 \mathrm{~Hz}$.

For a constant rate of stimulation, the pitch varies with the position of the electrodes in the scala tympani. For a pulse rate of $50 \mathrm{pps}$, the highest pitch estimate $(200$ mels) was obtained at electrode 7 , while the lowest pitch estimate ( 10 mels) was at electrode 1 . The lower pitch perceived with stimulation of electrode 9 at 50 pps was most likely due to its position in the region of the round window as this could result in the extra-cochlear spread of current to the internal auditory meatus and excitation of auditory nerve fibres with lower characteristic frequencies.

The same pitch could be produced by driving single electrodes with different pulse rates. From Fig. 4, it can be seen that the pitch estimate for a 50 pps stimulus at electrode 7 and the pitch estimates for 100 pps stimuli at three other electrodes $(3,6$ and 8$)$ are approximately the same.

Although the same pitch could be produced at different electrodes, the hearing sensations at different electrodes were consistently described by the subject as 'different sounds'. For example, the hearing sensations at electrodes 1 and 9 were described as 'dull sounds' at low pulse rates, while 'very sharp sounds' were reported when electrode 7 was driven with pulse rates in the same range. These informal observations suggested that dis- 
similar hearing sensations having the same subjective pitch could be produced at different electrodes.

What were these dissimilar sensations? Could they be utilized in the encoding of electrical stimuli? These questions led to the identification experiments to be described in the following section.

\section{(iv) Identification experiments}

Steady state electrical stimuli with pulse rates ranging from $50 \mathrm{pps}$ to $1,000 \mathrm{pps}$ were delivered to single electrodes. After the presentation of a stimulus, the patient was asked to sing or sound the hearing sensation produced by the stimulus.

For pulse rates above $150 \mathrm{pps}$, most of the stimuli were 'too high in pitch' for the patient to mimic as his voice range only goes up to an octave above middle $\mathrm{C}$ on the musical scale. For pulse rates ranging from $50 \mathrm{pps}$ to $125 \mathrm{pps}$, however, the subject was able to mimic the hearing sensations. With these informal experiments, it was soon discovered that the mimicked sounds appeared to possess distinct spectral colours. For example, a 100 pps stimulus at electrode 9 was mimicked as /a/ as in father, while the same stimulus at electrode 7 was mimicked as $/ \mathrm{i} /$ as in seat.

With these results in mind, the following identification experiment was designed to investigate further the nature of the phenomenon. Nine $75 \mathrm{pps}$ pulse trains, one for each electrode, were arranged in irregular order and presented to the patient with instructions to try to identify each hearing sensation as a speech sound, and to describe the pitch and sharpness of the sensation. The nine pulse trains were $200 \mathrm{~ms}$ in duration with rise-decay times of $50 \mathrm{~ms}$. Current levels were adjusted to provide a subjective judgement of medium loudness. The loudnesses were balanced across the nine electrodes. To familiarize the patient with the range of possible speech sounds, the patient was given a list of English phonemes with word examples, and was instructed to study the list at home with the help of his family.

The results of this identification experiment are summarized in Table I. The hearing sensations produced by the nine stimuli were described as mechanical. The identifications were consistent over four irregular presentations of the nine stimuli. Some confusions between $/ \varepsilon /$ and $/ I /$ were observed at electrode 2 .

From these results, it can be concluded that the patient was able to associate the hearing sensations produced by single electrodes with different vowel colours, or in more general terms, spectral colours. This association is, of course, not unique. The patient could very well have been instructed to identify the sensations as environmental sounds. However, the results obtained by the present technique should be directly applicable to the selection of a speech processing scheme that is acceptable to the patient.

The vowel colours identified at electrode 9 and electrodes 3 to 7 were dependent on the duration of the pulse trains. Informal experiments have 
TABLE I

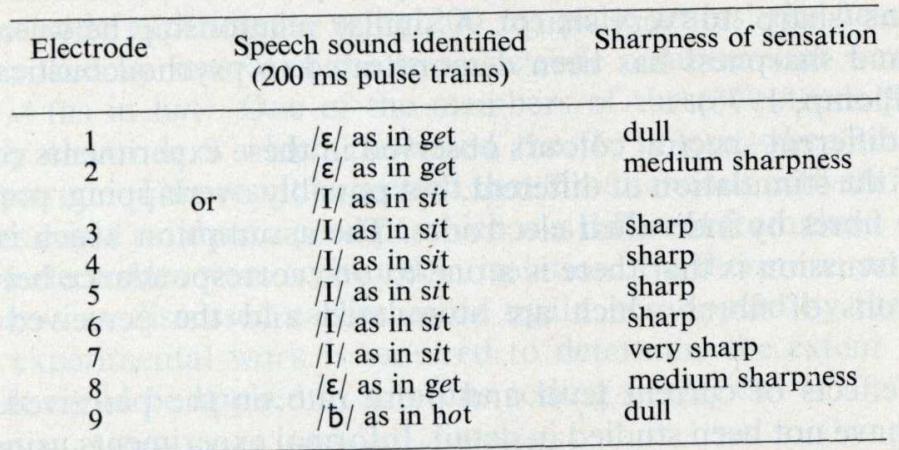

shown that, when the duration of the 75 pps pulse trains was increased to $400 \mathrm{~ms}$, an / $/ 2$ (as in cord) was identified at electrode 9, and an /i/ (as in seat) was identified at electrodes 3-7. The $|\varepsilon|$ at electrodes 1,2 and 8 , however, appeared to be independent of the duration. It should be noted that, in spoken English, the formant characteristics of $/ \mathbf{b} /$ are closely approximated by those of $/ \mathrm{\rho} /$, and those of $/ \mathrm{I} /$ by /i/ (Ainswurth, 1976). Furthermore, the durations for $/ \mathrm{b} /$ and $/ \mathrm{I} /$ were found to be approximately half of those for $/ 2 /$ and $/ \mathrm{i} /$ (Bernard, 1970). It is possible that the electrically evoked sensation at electrode 9 was characterized by a vowel colour which was similar to $/ \mathrm{b} /$ and $/ \mathrm{\partial} /$, and the identification was based on the duration of the sensation. The distinction between $/ \mathrm{I} /$ and $/ \mathrm{i} /$ may be similarly cued.

For the purpose of developing an appropriate system of stimulus coding, it is important to derive equivalent acoustical signals that would produce the spectral colours $(/ \mathrm{b} /, / \mathrm{\rho} /, \mid \varepsilon /, / \mathrm{I} /$ and $/ \mathrm{i} /)$ reported by the patient. As a first approximation, these equivalent acoustical signals can be derived from results obtained in single-formant synthetic vowel studies. Delattre et al. (1952), have shown that single-formant synthetic vowels with formant frequencies at $720 \mathrm{~Hz}, 2,160 \mathrm{~Hz}$ and $3,000 \mathrm{~Hz}$ were identified respectively as having vowel colours corresponding to $/ \nu /, \mid \varepsilon /$ and $/ \mathrm{i} /$. For formant frequencies above $3,000 \mathrm{~Hz}$, the single-formant synthetic vowels were always identified as $/ \mathrm{i} /$. Single-formant synthetic signals for the short vowels $/ \mathrm{b} /$ and $/ \mathbf{I} /$ were not studied by Delattre et al. (1952). However, as discussed earlier, there are only slight differences between the formant characteristics for $/ \mathrm{b} /$ and $/ \mathrm{\nu}$, and between those for $/ \mathrm{I} /$ and $/ \mathrm{i} /$. For this reason, it may be assumed that formant frequencies for the single-formant synthetic vowels for $/ 0 /$ and $/ \mathrm{i} /$ are close approximations to those for $/ \mathrm{b} /$ and $/ \mathbf{I} /$. If these approximations are appropriate, the spectral colours produced here by electrical stimulation may be approximately equivalent to those produced by acoustical signals with their spectral emphases centred at frequencies ranging from $720 \mathrm{~Hz}$ to above $3,000 \mathrm{~Hz}$.

The reported spectral colours were characterized by a specific sharpness, a component of the timbre attribute of a hearing sensation (Plomp, 1976). The low frequency $/ \mathrm{b} /, / \partial /$ and $/ \varepsilon /$ colours at electrodes 1 and 9 were 
described as 'dull', while the high frequency /I/ and /i/ colours were described as 'sharp' to 'very sharp'. A similar relationship between spectral colour and sharpness has been demonstrated in psychoacoustical experiments (Plomp, 1976).

The different spectral colours observed in these experiments could be a result of the stimulation of different, but possibly overlapping, populations of nerve fibres by individual electrodes. The assumption which is implicit in this discussion is that there is a one-to-one correspondence between the populations of fibres which are stimulated and the perceived spectral colours.

The effects of current level and pulse rate on the perceived spectral colours have not been studied in detail. Informal experiments using $400 \mathrm{~ms}$ pulse trains have shown that the $/ 0 /$ colour at electrode 9 could become an /a/ colour with an increase in current level or pulse rate.

The effect of current level on the perceived spectral colours at electrode 9 could be a result of current spread to nerve fibres excited by other electrodes, and an 'averaging' process adopted by the patient. As a result of this spread, other spectral colours would be perceived in addition to the colour evoked by stimulating fibres normally responsive to electrode 9 at lower current levels. Assuming that the subject based his judgment on a weighted average of the evoked spectral colours, a spread of current from electrode 9 to other electrodes possessing the $|\varepsilon|$ and $/ i /$ colours would result in a colour different from $/ 0 /$ an $/ a /$ in this case. In this regard Delattre et al. (1952) have shown that a single- formant synthetic vowel with its spectral emphasis centred at $1,200 \mathrm{~Hz}$ was identified as having the /a/ colour, while synthetic vowels with their spectral emphases centred at $720 \mathrm{~Hz}$ and $2,160 \mathrm{~Hz}$ were identified respectively as $/ \supset /$ and $/ \varepsilon /$. The fact that a synthetic /a/ colour has its spectral centre at an intermediate frequency between the frequencies for synthetic $|\rho|$ and $|\varepsilon|$ supports the notion of an averaging process.

A change in pulse rate also produced a change in spectral colours. This was seen when stimulating electrode 9 . In this case the vowel percept changed from $/ 2 /$ to $/ a /$ with an increase in rate. This was also associated with an increase in pitch (Fig. 4).

\section{(B) Two-electrode studies}

(i) Stimulation using the same pulse rate

Identification experiments as described in section (A) were performed with two electrodes driven at the same pulse rate. A pulse rate of $75 \mathrm{pps}$ was used. The pulse trains were $200 \mathrm{~ms}$ in duration with rise-decay times of $50 \mathrm{~ms}$. The current levels of the pulse trains were identical to those used in the experiments described in section (A). 36 stimuli corresponding to all the possible 36 combinations of electrode-pairs were presented in irregular order, and the patient was asked to identify each hearing sensation as a speech sound. 


\section{University Library}

\section{- M M N E R VA A gateway to Melbourne's research publications}

Minerva Access is the Institutional Repository of The University of Melbourne

Author/s:

Tong, Y. C.;Black, R. C.;Clark, Graeme M.;Forster, I. C.;Millar, J. B.;O'Loughlin, B. J.;Patrick, J. F.

Title:

A preliminary report on a multiple-channel cochlear implant operation

Date:

1979

\section{Citation:}

Tong, Y. C., Black, R. C., Clark, G. M., Forster, I. C., Millar, J. B., O'Loughlin, B. J., et al. (1979). A preliminary report on a multiple-channel cochlear implant operation. Journal of Laryngology and Otology, 93(7), 679-695.

Persistent Link:

http://hdl.handle.net/11343/28720 